\title{
The XMediaBox: Sensemaking through the Use of Knowledge Lenses
}

\author{
Aba-Sah Dadzie ${ }^{1}$, José Iria ${ }^{1}$, Daniela Petrelli ${ }^{2}$, and Lei Xia ${ }^{1}$ \\ 1 Dept. of Computer Science \\ 2 Dept. of Information Studies \\ The University of Sheffield \\ \{a.dadzie, j.iria,d.petrelli,l.xia\}@sheffield.ac.uk
}

\begin{abstract}
Sensemaking is the process of analysing complex situations in order to make informed decisions. Semantic Web technology can be effectively used to create new sensemaking systems that focus on concepts and knowledge instead of documents. We demonstrate how this is achieved using information extraction to acquire knowledge and create a semantic repository that can then be semantically searched. A domain ontology is used to support the creation of an analysis tree; the semantic visualisation enables knowledge discovery, a core aspect of sensemaking.
\end{abstract}

Keywords: Semantic web, Knowledge acquisition, Semantic visualisation, Sensemaking.

\section{Background}

Sensemaking is the process of analysing complex and uncertain situations in order to take informed decisions [1. It requires knowledge workers to find relevant information and formulate hypotheses based on the knowledge contained, test the hypotheses and revise the effectiveness of the decisions adopted. While some systems support only document manipulation in sensemaking (e.g., [2]), the X-Media project [3] uses Semantic Web (SW) technology and ontology-based visualisation to enable abstraction from the low level of multiple instances and more easily compose a conceptual model of a situation. Knowledge acquisition and retrieval, and visualisation are basic components of semantic sensemaking.

Knowledge acquisition (KA) transforms information into knowledge via classification and extraction. While information is heterogeneous and dispersed, coming from many sources, e.g., text documents, images, tables, etc., knowledge is homogeneous and instantiates concepts and relations captured in domain ontologies. In knowledge retrieval, users search knowledge repositories to find those missing elements that would improve their understanding of the current situation. Knowledge visualisation supports visual thinking by explicitly laying out the retrieved knowledge, information and personal reflections.

We demonstrate the steps we follow during analysis using the XMediaBox, developed as part of X-Media, using a non-restricted dataset. The XMediaBox has also been evaluated with experts in a realistic, knowledge-intensive setting: aerospace engineers working on gas turbine engines [4]. 


\section{Semantic Web for Sensemaking}

We make use of SW technologies in three ways, by: i) capturing knowledge from archive documents via automated KA from multimedia; ii) searching archive information via hybrid search (semantic and keyword-based); and iii) visualising knowledge-in-context to support visual thinking.

\subsection{Capturing Knowledge}

A wealth of information is "hidden" within unstructured text, image, and mixedmedia content. Information Extraction (IE) from text produces a semantic representation of the information (entities and relations) expressed in the text in natural language. Analogously, Content Based Image Retrieval systems produce a semantic representation of image content. Our applications represent information using ontologies; in particular, we use the Core Ontology of Multimedia (COMM) [5] to represent the knowledge captured from multimedia documents.

We use state-of-the-art algorithms from the IE and IR (information retrieval) literature in addition to rule- and machine learning-based approaches (Saxon [6], T-Rex [7) to extract knowledge from single and multiple media documents. We perform entity recognition and relation extraction from text and regionof-interest classification from images to populate the knowledge repository (8] details our approach). Fig. 1 shows an example of the semi-structured, multimedia documents analysed in X-Media, with (form) fields mapping to concepts in the domain ontology and images whose content may be described in the text.

\subsection{Searching Knowledge}

The KA step creates a knowledge base (KB) that the user can search using specific tools. We will demonstrate a hybrid search engine that combines ontologyand keyword-based searching to increase the effectiveness of retrieval [9. The knowledge retrieved and associated documents may be inspected by the user, who decides relevance to the current analysis. Adding the knowledge retrieved into the ongoing sensemaking scenario enriches the metadata attached to it, and as a result, the knowledge captured in the KB. This also aids data categorisation, leading to more accurate retrieval across different media and document types.

\subsection{Visualising Knowledge}

Information visualisation provides effective support for intuitive analysis [10 and understanding of ontology structure [11 and ontology-based information. In XMedia we go a step further and provide multiple ways of visualising knowledge in order to support better understanding of complex scenarios. We have developed Knowledge Lenses, alternative views on the underlying data that support different levels of abstraction. Each knowledge lens has a different focus: the interconnections between knowledge in documents, human understanding (via notes), or the knowledge in the semantic repository. The semantics behind the data enable a smooth transfer from document to knowledge to personal notes. 


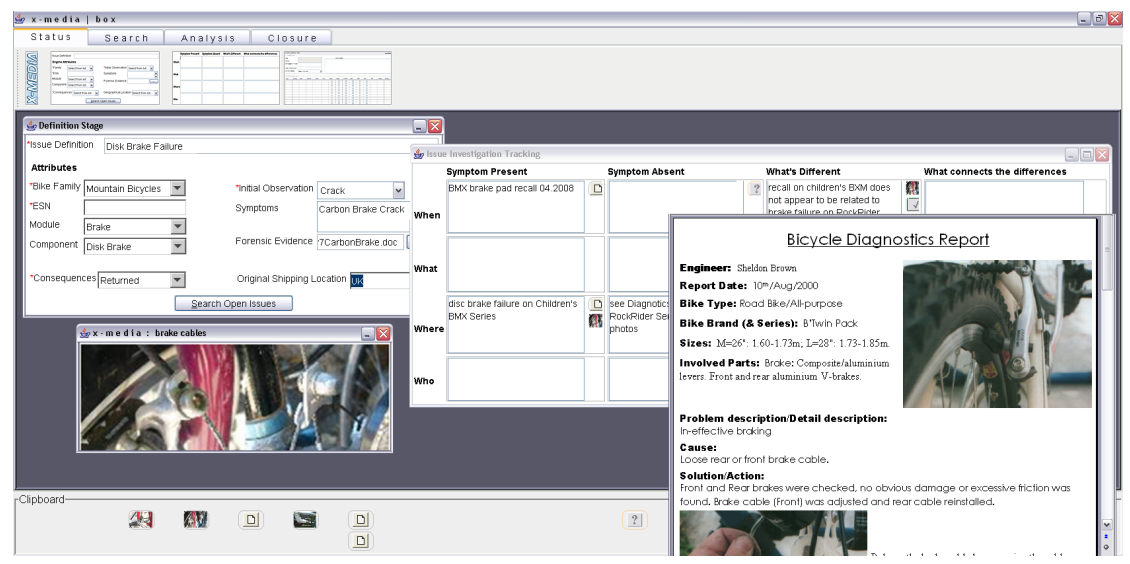

Fig. 1. A definition form in the XMediaBox (top, left) maps form fields to relevant concepts in the domain ontology. The interactive table (top, right) contains users' thoughts linked to evidence. The panel at the bottom is used to bookmark and share knowledge objects. Two examples of the data referenced within the system are shown.

\section{Sensemaking via Knowledge Lenses}

The sensemaking process within the XMediaBox is articulated in several steps, each providing a different view on the current situation and related information. Each step corresponds to a tab on the user interface (see the top of Fig 1: status, search, analysis and closure). The sensemaking process may span several months; however it always begins by defining the purpose of the investigation. A definition form contextualises the issue using selected domain ontology concepts. The information input by the user initialises a search of legacy corpora and other pertinent repositories to identify information relevant to the current case. The documents retrieved may be inspected and, if found to be relevant, bookmarked for future use, for e.g., as evidence to support a hypothesis.

As the sensemaking progresses evidence is collected and users' understanding develops. The status of the investigation is recorded using interactive tables (see Fig. 1), contextualising the implicit knowledge embedded in the documents using free text and semantic annotation via ontologies. The tables summarise the knowledge exploration, supporting sharing with other knowledge workers.

While search is an activity carried out only when the user needs to collect new evidence, analysis, which is the core of sensemaking, is sustained throughout the process. User understanding is built up during the systematic analysis of evidence and counter-evidence, and on the evaluation of which hypotheses explain the phenomena observed. The XMediaBox captures this rationalisation process in the interactive analysis tree (see top, Fig. 2) that helps users formulate new and analyse existing hypotheses. The system supports users in building the analysis tree from suggestions in the domain ontology, focusing on the relations hasCause (with inverse hasEffect), and subpart and is $A$ relations between physical objects. 


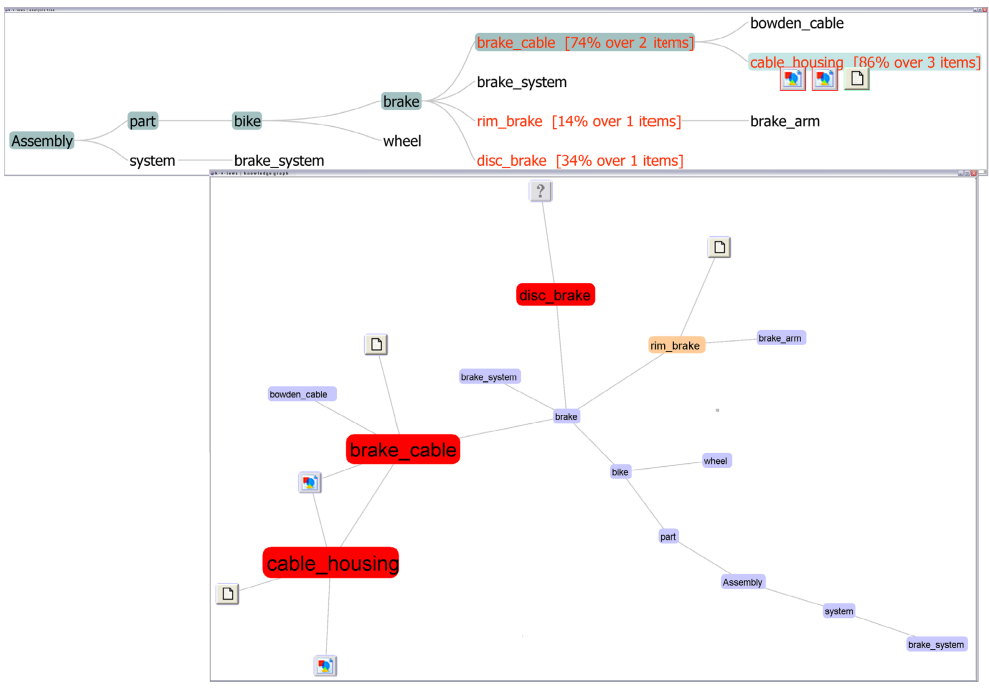

Fig. 2. Two knowledge lenses based on a domain ontology. The knowledge tree (top) provides structured visual analysis, and the knowledge graph (bottom) clusters hypotheses with evidence in common, simultaneously highlighting outliers. Attaching evidence to nodes tags them with the corresponding hypotheses, enriching the metadata and leading to more effective knowledge creation, categorisation, retrieval and reuse. [The modules were built using the prefuse visualisation toolkit (http://prefuse.org)].

Relevant documents, snippets of evidence and comments retrieved from the $\mathrm{KB}$ or created within the system may be attached to each (cause/hypothesis) node in the tree during the sensemaking process, creating an individual perspective on the hypotheses being investigated and the resulting knowledge space. The knowledge lens thus created supports pattern spotting, helping users determine which branch in the investigation contains the more valid hypothesis and which others need further investigation. Attaching the multimedia document in Fig. 2 to the node brake_cable indicates that it contains evidence that the hypothesis "failure of the brake cable" may be the root cause of the issue. Extracting a snippet that links the concept component to disc_brake with the relation removed (with a record of its provenance), is equivalent to manually annotating the document content. Both actions update the metadata in the knowledge repository; subsequent searches on the ontology concepts will retrieve this document.

While the knowledge tree provides a structured view of the knowledge space for the current analysis, the knowledge graph provides a more complete overview of the relationships between the hypotheses (concepts of interest) and the knowledge and annotated evidence. The knowledge graph serves as an alternative, less restricted view on the knowledge space, showing interconnections between evidence that may not be easily identified otherwise. It further supports the discovery of existing, but not yet identified knowledge during exploratory analysis, by providing a means for browsing the contents of the knowledge repository.

A prototype of the XMediaBox has been evaluated by 12 aerospace engineers during a simulation of a sensemaking activity using a grounded, domain-specific 
scenario [4. Documents fed into the knowledge capture phase used multiple formats: text only, images only, or multimedia - both text and images. The documents formed part of a very large company archive, processed using the KA techniques summarised. Guided by ontology-based suggestions, the engineers extended an analysis tree, populated the tree with the factual evidence retrieved, and recorded their understanding of the knowledge space created. The evaluation showed that Semantic Web technology effectively supports different levels of abstraction: table summaries, tree analysis and exploratory graphs, shifting the sensemaking activity from document manipulation and management to a higher conceptual level, that of knowledge management and discovery.

\section{Acknowledgements}

The research described in this paper is funded by the X-Media project, sponsored by the European Commission as part of the Information Society Technologies (IST) programme under EC grant number ISTFP6-026978. Thanks also to Fabio Ciravegna for the invaluable discussions and contributions to the paper.

\section{References}

1. Klein, G., Moon, B., Hoffman, R.: Making sense of sensemaking 1: Alternative perspectives. IEEE Intelligent Systems, 70-73 (2006)

2. Wright, W., Schroh, D., Proulx, P., Skaburskis, A., Cort, B.: The Sandbox for analysis: concepts and evaluation. In: Proceedings of the SIGCHI conference on Human Factors in Computing Systems, pp. 801-810 (2006)

3. The X-Media Project, http://www.x-media-project.org

4. Dadzie, A.-S., Lanfranchi, V., Petrelli, D.: Seeing is believing: Linking data with knowledge. Information Visualization (in press)

5. Arndt, R., Troncy, R., Staab, S., Hardman, L., Vacura, M.: Comm: Designing a well-founded multimedia ontology for the web. In: Aberer, K., Choi, K.-S., Noy, N., Allemang, D., Lee, K.-I., Nixon, L., Golbeck, J., Mika, P., Maynard, D., Mizoguchi, R., Schreiber, G., Cudré-Mauroux, P. (eds.) ASWC 2007 and ISWC 2007. LNCS, vol. 4825, pp. 30-43. Springer, Heidelberg (2007)

6. Greenwood, M., Iria, J., Ciravegna, F.: Saxon: An extensible multimedia annotator. In: 6th International Conference on Language Resources and Evaluation (2008)

7. Iria, J.: T-Rex: A flexible relation extraction framework. In: 8th Annual Colloquium for the UK Special Interest Group for Computational Linguistics (2005)

8. Dadzie, A.S., Bhagdev, R., Chakravarthy, A., Chapman, S., Iria, J., Lanfranchi, V., Magalhães, J., Petrelli, D., Ciravegna, F.: Applying semantic web technologies to knowledge sharing in aerospace engineering. Intelligent Manufacturing (2008)

9. Bhagdev, R., Chapman, S., Ciravegna, F., Lanfranchi, V., Petrelli, D.: Hybrid search: Effectively combining keywords and semantic searches. In: Bechhofer, S., Hauswirth, M., Hoffmann, J., Koubarakis, M. (eds.) ESWC 2008. LNCS, vol. 5021, pp. 554-568. Springer, Heidelberg (2008)

10. Keim, D., Andrienko, G., Fekete, J.D., Görg, C., Kohlhammer, J., Melançon, G.: Visual analytics: Definition, process, and challenges. In: Kerren, A., Stasko, J.T., Fekete, J.D., North, C. (eds.) Information Visualization: Human-Centered Issues in Visual Representation, Interaction, pp. 154-175. Springer, Heidelberg (2008)

11. Geroimenko, V., Chen, C. (eds.): Visualizing the Semantic Web: XML-based Internet and Information Visualization, 2nd edn. Springer, Heidelberg (2006) 